\title{
REVIEW
}

\section{Community-acquired pneumonia as an emergency condition}

\section{Purpose of review}

Despite the improvements in its management, community-acquired pneumonia (CAP) still exhibits high global morbidity and mortality rates, especially in elderly patients. This review focuses on the most recent findings on the epidemiology, cause, diagnosis and management of CAP.

\section{Recent findings}

There is consistent evidence that the trend in CAP mortality has declined over time. However, the mortality of pneumococcal CAP has not changed in the last two decades, with an increase in the rate of hospitalization and more severe forms of CAP. Streptococcus pneumoniae remains the most frequent cause of CAP in all settings, age groups and regardless of comorbidities. However, the implementation of molecular diagnostic tests in the last years has identified respiratory viruses as a common cause of CAP too. The emergency of multidrug-resistance pathogens is a worldwide concern. An improvement in our ability to promptly identify the causative cause of CAP is required in order to provide pathogen-directed antibiotic therapy, improve antibiotic stewardship programs and implement appropriate vaccine strategies.

\section{Summary}

It is time to apply all the knowledge generated in the last decade in order to optimize the management of CAP.

\section{Keywords}

community-acquired pneumonia, epidemiology, management, microbial cause

AQ4

community-acquired pneumonia (CAP) is an emergency condition with high morbidity and mortality [1-3]. The incidence of CAP continues to rise, especially among elderly and immunocompromised patients $[3,4]$. Pneumonia is the most common infection leading to sepsis $[5,6]$. A recent study reported the association of four comorbidities (diabetes mellitus, dementia, chronic heart failure and coronary heart disease) on the development of sepsis in CAP patients [7]. Approximately 65\% of elderly patients hospitalized with CAP have two or more comorbidities, thus experiencing a higher risk of being affected by sepsis $[4,8]$.

Severe CAP is frequently complicated by pulmonary and extra-pulmonary complications, including sepsis, septic shock, acute respiratory distress syndrome and acute cardiac events, thus resulting in a significant increase of mortality and need for ICU admission [4,9-13]. Although there is no general consensus on its definition, the most accepted criteria to address severe CAP are based on the 2007
Infectious Diseases Society of America/American Thoracic Society consensus guidelines on the management of CAP in adults [1]. Severe CAP is defined by the presence of two major criteria: severe acute respiratory failure requiring invasive mechanical ventilation and/or septic shock. Several minor criteria requiring high-intensity monitoring and treatment have been proposed for severe CAP diagnosis too [14].

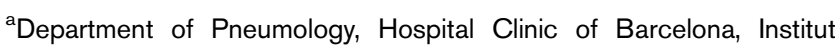
d'Investigacions Biomèdiques August Pi i Sunyer (IDIBAPS), University of Barcelona (UB) - SGR 911- Ciber de Enfermedades Respiratorias (Ciberes) Barcelona, Barcelona, Spain, ${ }^{\mathrm{b}}$ Department of Anesthesiology and Intensive Care Medicine, Fondazione Policlinico Universitario A. Gemelli, Università Cattolica del Sacro Cuore, Rome, Italy and ${ }^{\mathrm{C}}$ InfecInfectious Disease Department, Hospital Clinic of Barcelona, Barcelona, Spain

Correspondence to Antoni Torres, Department of Pulmonary Medicine, Hospital Clinic of Barcelona, C/ Villarroel 170, 08036 Barcelona, Spain. Tel: +34 93227 5779; fax: +34 93227 9813; e-mail: atorres@clinic.cat

Curr Opin Crit Care 2018, 24:000-000

DOI:10.1097/MCC.0000000000000550 


\section{KEY POINTS}

- The incidence of CAP continues to rise, especially among elderly and immunocompromised patients.

- Early recognition and management of severe CAP is of pivotal importance in order to reduce mortality in critically ill patients.

- Appropriate site of care decisions, based on clinical assessment and supported by prognostic tools, can improve patients' outcomes.

- Risk factors for MDR pathogens should be recognized in order to initiate an adequate empiric antibiotic therapy.

- Inflammatory biomarkers may be a complementary prognostic tool for the management of CAP patients.

- There is lack of evidence regarding the utility of PCT in antibiotic stewardship.

- Empiric antibiotic therapy should be based on international and local guidelines, as this is associated with improved outcomes.

- The use of corticosteroids should be considered in severe CAP cases with high inflammatory response (serum levels of C-reactive protein $\geq 15 \mathrm{mg} / \mathrm{dl}$ ).

CAP management in the emergency department affects the final outcome of the infection. The following interventions have been related to improved outcomes; prompt diagnosis through a chest X-ray within the first $4 \mathrm{~h}$ of presentation; early appropriate antimicrobial therapy, covering multidrug resistant pathogens when suspected; early identification of patients requiring respiratory and hemodynamic support and thus ICU admission; mortality risk assessment using severity scores have been related with improved outcomes [15].

This review summarizes the most recent findings regarding CAP cause, risk factors, biomarkers and therapy.

\section{WHY IS COMMUNITY-ACQUIRED PNEUMONIA SEVERITY ASSESSMENT IMPORTANT?}

After the initial diagnosis of CAP, the biggest challenge in the emergency department is to promptly recognize patients who might develop respiratory failure and multiple organ dysfunctions. Patients requiring mechanical ventilation or vasopressor support should be admitted to ICU as soon as possible, as delays in ICU admission have been related to worse outcomes [16-18].

Scoring systems such as the Pneumonia Severity Index (PSI) [19] and the CURB-65 [14] (confusion, urea, respiratory rate, blood pressure and age $>65$ years) predict short-term mortality and have been developed to specifically support clinicians in the decision to admit patients to the hospital; however, their ability to predict the need for ICU admission has not been demonstrated.

Recently, a Spanish study [20"'] compared the clinical usefulness of the quick Sequential (Sepsisrelated) Organ Failure Assessment (qSOFA), the criteria for Systemic Inflammatory Response Syndrome (SIRS), CURB-65, CRB (confusion, respiratory rate and blood pressure), modified Sepsis Organ Failure Assessment (mSOFA) and PSI in a retrospective cohort of 6874 patients with CAP. The authors concluded that CRB and qSOFA outperformed SIRS, whereas PSI was the best predictor of mortality.

The ATS/IDSA 2007 major-minor criteria [1] and the SMART-COP [21] (systolic blood pressure, multilobar chest radiograph involvement, albumin, respiratory rate, tachycardia, confusion, oxygenation and arterial $\mathrm{pH}$ ) are the scores most commonly used to predict ICU admission.

The IDSA/ATS major (need for mechanical ventilation or septic shock) and minor (respiratory rate $\geq 30$ breaths/min, $\mathrm{PaO} 2 / \mathrm{FiO} 2$ ratio $\leq 250$, multilobar infiltrates, confusion or disorientation, blood urea nitrogen $\geq 20 \mathrm{mg} / \mathrm{dl}$, leukocyte count $<4 \times 10^{9}$ cells/ 1 , platelets count $<100 \times 10^{9}$ cells $/$, temperature $<36^{\circ} \mathrm{C}$ and hypotension requiring aggressive fluid resuscitation) criteria have been proposed to identify patients with severe CAP requiring ICU admission. Minor criteria are easy to use and have shown high specificity (91.7\%) in predicting ICU admission and the need for intensive respiratory or vasopressor support $[17,22]$. Even in the absence of major criteria, the presence of at least three minor criteria has been associated with complications and a high 30-day mortality risk $[22,23]$.

SMART-COP [21] has been validated in several studies $[24,25]$. It is based on eight parameters: multilobar involvement (1 point), low systolic blood pressure ( 2 points), high respiratory rate (1 point), low albumin level (1 point), tachycardia (1 point), confusion (1 point), poor oxygenation (age adjusted, 2 points) and low arterial $\mathrm{pH}$ (2 points). The Australian CAP Study (ACAPS) found that a SMART-COP score of at least 3 points identified the majority of patients who received intensive respiratory or vasopressor support. Its accuracy was higher than PSI classes IV and V and CURB65 group 3. SMART-COP was accurate both for patients who were admitted to ICU directly from the emergency department (sensitivity, 98\%) and for those who were initially admitted to the general ward before their condition deteriorated (sensitivity, $84 \%)$. 
Despite these results, both scores present several limitations. The ATS/IDSA criteria do not take into account the criteria 'age'. The SMART-COP score cutoff for age is 50 years, thus limiting its applicability in elderly patients. The omission of other AQ5 (CD teria such as COPD, immunosuppression, patient uependency and other social factors limits the accuracy of the scores.

In conclusion, the experience and judgment of clinicians in assessing patient severity remain vital in the clinical management. We suggest to calculate PSI or CURB65 after the initial diagnosis of CAP in order to decide if the patient requires hospital admission.

A second score (ATS/IDSA mayor and minor criteria, SMART-COP, etc.) should be use to evaluate the necessity for ICU admission.

\section{WHAT IS THE ROLE OF BIOMARKERS IN COMMUNITY-ACQUIRED PNEUMONIA?}

Biomarkers provide reliable information about the host response to infection as well as the response to pharmacological therapy. Unfortunately, the heterogenic immunological and inflammatory profiles of CAP patients make the universal use of biomarkers difficult $\left[26^{*}, 27\right]$. Biomarkers such as the C-reactive protein (CRP), procalcitonin (PCT), lymphocytes, soluble triggering receptor expressed on myeloid cells- 1 , proadrenomedullin, copeptin and soluble form of urokinase-type plasminogen activator receptor have been studied and some of them validated [26",28-34]. The biomarkers mostly used in the clinical practice are CRP, PCT and more recently lymphocytes.

\section{BIOMARKER-GUIDED ANTIBIOTIC THERAPY}

CRP is a major acute phase protein synthesized by hepatocytes. CRP is stimulated by interleukin 6 and 8 (IL-6 and IL-8), mainly produced by macrophages in response to any type of inflammation, including bacterial and viral infection. The protein release starts $4-6 \mathrm{~h}$ after the beginning of the inflammation, with a peak around $36-48 \mathrm{~h}$. The plasma halflife is about $7-10 \mathrm{~h}$. CRP is not specific for the diagnosis of pneumonia as its levels can be elevated in other clinical conditions such as neoplasia or autoimmune diseases [35].

PCT is a calcitonin precursor peptide usually synthesized in the thyroid gland. It increases during inflammatory and infectious diseases. In healthy individuals, it is almost undetectable or lower than $0.05 \mathrm{ng} / \mathrm{ml}$. PCT has been demonstrated to be useful to differentiate pneumonia from other clinical conditions [35].
Recently, in a multicenter prospective surveillance study of 1735 adults hospitalized with CAP [36"], no PCT threshold was found to specifically discriminate between viral and bacterial pathogens. However, higher values of PCT strongly correlated with an increased probability of bacterial pathogens, particularly typical bacteria (Fig. 1).

Interestingly, a recent multicenter trial conducted in 14 hospitals from United States investigated the effect of PCT-guided antibiotic use on the treatment of suspected lower respiratory tract infections [37]. Data of 1656 patients (826 randomly assigned to the PCT group and 830 to the usual-care group) were analyzed. In both groups, PCT-level tier was associated with the decision to prescribe antibiotics in the emergency department. There was no significant difference between the two groups in either antibiotic-days or the proportion of adverse outcomes within 30 days.

Similarly, Chu et al. [38] investigated the PCT use in critically ill patients with sepsis in a real-world setting where the main site of infection was pneumonia (accounting for $35 \%$ of the study population). PCT use did not improve antibiotic use or antibiotic-associated outcomes on average; conversely, PCT use was associated with an increased incidence of Clostridium difficile infections.

The results of the two above mentioned studies may be interpreted considering the partially understood PCT biology and physiology and the variability of PCT levels in some situations such as acute kidney injury or renal replacement therapies. These results contrast with the findings of a previous systematic review and meta-analysis [39"]. PCT use to guide initiation and duration of antibiotic treatment in acute respiratory tract infections resulted in lower risk of mortality, lower antibiotic consumption and lower risk of antibiotic-related side-effects.

Despite the promising value of CRP and PCT, there are still several limitations for their use in the daily clinical practice. Clinical judgment, severity scores and biomarkers should help in the diagnosis of CAP and in the beginning of the empiric antibiotic therapy.

\section{BIOMARKERS FOR COMMUNITY- ACQUIRED PNEUMONIA PROGNOSIS}

Several studies reported that biomarkers can also be used to predict clinical outcomes, complications such as bacteremia and mortality in patients affected by CAP $[29,35]$.

The recent study by McCluskey et al. [40"] investigating the use of PCT to predict mortality, ICU admission and bacteremia in 317 patients with pneumonia (191 CAP and 126 HCAP) reported that 


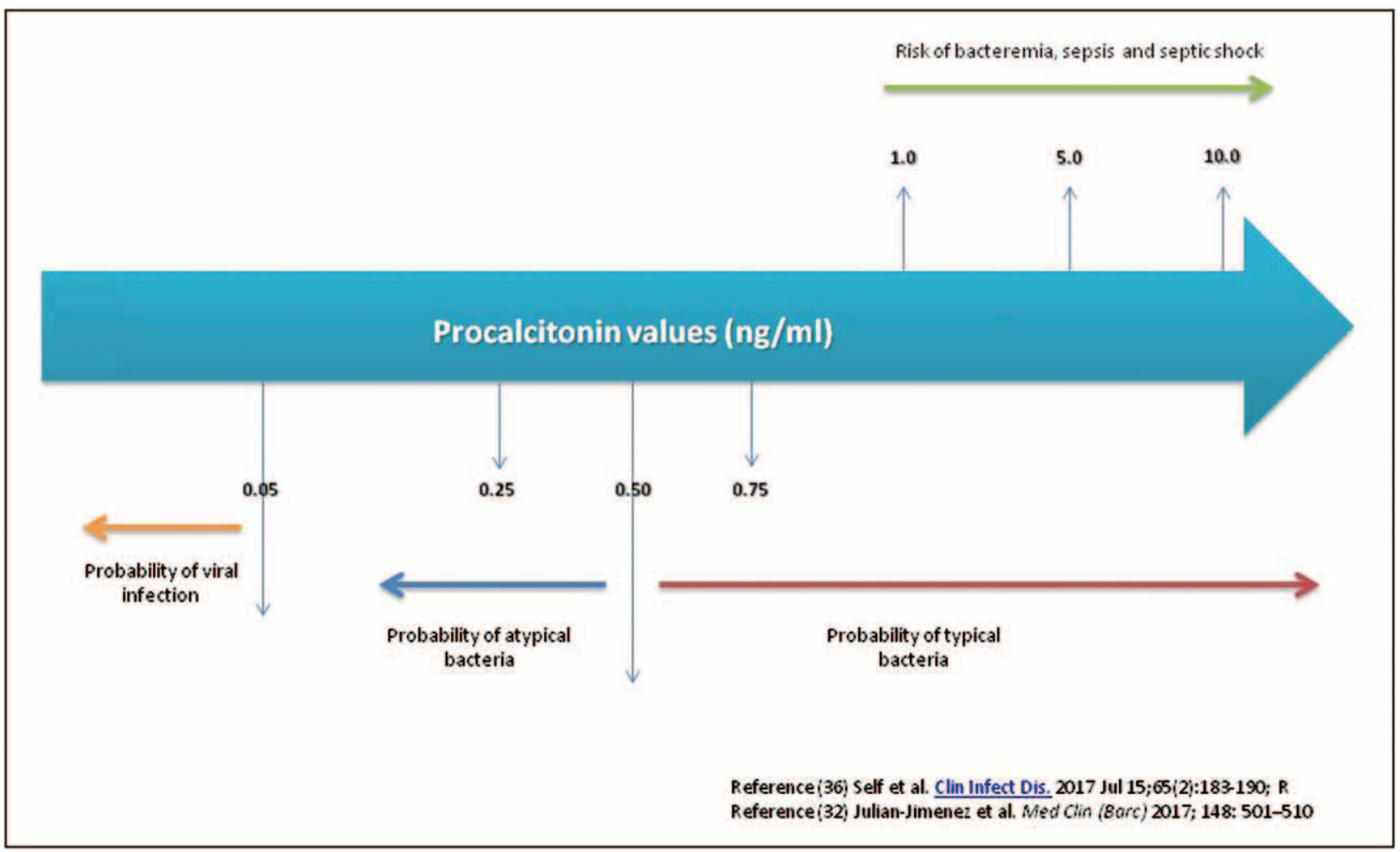

FIGURE 1. Procalcitonin: microbial cause and prognostic outcomes in CAP. CAP, community-acquired pneumonia.

serial PCT measurements improve the performance of clinical scores (PSI) to predict bacteremia and the necessity for ICU-level care nevertheless without predicting mortality.

Zhou et al. [29] investigated the risk stratification and prognostic prediction value of PCT and clinical severity scores (CURB65, PSI, SOFA and qSOFA) in patients with CAP admitted at the emergency department. The authors reported that PCT was a predictor of severe CAP. Compared to other combinations, the combination of PCT and SOFA score achieved the highest superiority in predicting not only severe CAP but also 28-day mortality.

A study from South Korea investigated the ability of CRP, PCT and various clinical risk scales (PSI, ATS/IDSA and CURB65) to predict 28-day mortality in CAP patients. PCT was a predictor of short-term mortality in CAP patients. Consequently, the author suggested that the use of CRP and/or PCT could significantly improve the performance of the PSI and the IDAS/ATS score [41].

Recently, some studies examined the role of peripheral blood lymphocytes count as prognostic predictors in CAP. A study from Spain [26"] investigated the association between neutrophil and lymphocyte counts and the mortality risk at 30-days post hospital admission in CAP patients. The presence of lymphopenia conferred an increased risk of mortality ('lymphopenic CAP profile'); furthermore, the addition of the lymphocyte count to the CURB65 improved the ability to predict 30-day mortality.

Interestingly, a study from China [42] found that the combination of $\mathrm{PO}_{2} / \mathrm{FiO}_{2}$ and lymphocyte counts predicted mortality and ICU admission in hospitalized patients with influenza pneumonia. Particularly, when $\mathrm{PO}_{2} / \mathrm{FiO}_{2}$ is less than 250 or peripheral blood lymphocyte count is less than $0.8 \times 10^{9} / \mathrm{l}$, great attention should be paid for the possibility of severe influenza pneumonia.

Some studies proposed the neutrophil to lymphocyte ratio (NLR) as simple and inexpensive indicators of systemic inflammation and infection. An Italian study [43] explored the performance of the NLR to predict prognosis in a cohort of 195 elderly adults with CAP. NLR predicted 30-day mortality and performed better than PSI, CURB65, CRP and white cell count to predict prognosis in the studied population. The authors recommended early discharge for CAP patients with NLR less than 11.12, short-term in-hospital care for CAP patients with NLR between 11.12 and 13.4, middle-term hospitalization for CAP patients with NLR between 13.4 and 28.3 and ICU admission for those with NLR at least 28.3.

The role of lymphocytes has also been explored in a recent Spain study in $160 \mathrm{HIV}$ patients with CAP [44]. The authors reported that red blood cell distribution and lymphocytes were the most useful 
predictors of disease severity in HIV CAP patients requiring ICU admission.

\section{THE IMPORTANCE TO IMPLEMENT A POINT-OF-CARE TESTING IN COMMUNITY- ACQUIRED PNEUMONIA}

Point-of-care testing (POCT) tools refer to etiologic test methods (e.g. viral respiratory panels), which can be used very close to the patient's treatment site, thus allowing faster CAP etiological diagnosis, early antimicrobial treatment and prompt therapy adjustments. An RCT by Brendish et al. [45"'] investigated the effect of routine POCT on a broad range of clinical outcomes (including antibiotic use) in adults presenting to the acute medical unit or to the emergency department with acute respiratory illnesses. Patients were allocated in the POCT group (a rapid molecular test for 15 respiratory viruses was performed with results available in $2-3 \mathrm{~h}$ ) or in the routine clinical care group. POCT did not reduce the proportion of patients treated with antibiotics. The authors explained that the result could be related to the administration of antibiotics, before test results were available, to a great proportion of patients in the POCT group. Interestingly, $91 \%$ of patients in the POCT group diagnosed with influenza virus received neuraminidase inhibitors compared with $65 \%$ of patients in the control group. The prompt initiation of neuraminidase inhibitor therapy in patients with influenza virus could have improved survival and reduced complications such as bacterial pneumonia. Other potential benefits of POCT in CAP caused by respiratory viruses included reduction of unnecessary antibiotic use, improved use of directed antiviral therapy for influenza virus, early identification of patients requiring isolation and reduction in hospital length of stay.

\section{WHAT ARE THE MAIN RISK FACTORS FOR MULTIDRUG-RESISTANT PATHOGENS?}

Although several bacteria and respiratory viruses are responsible of CAP, Streptococcus pneumoniae (Pneumococcus) remains the most common pathogen that causes it. Hoverer, approximately $6 \%$ of CAPs are caused by multidrug-resistant (MDR) pathogens, with Pseudomonas aeruginosa and methicillin-resistant Staphylococcus aureus (MRSA) being the most frequently reported $[46,47]$.

Because the antibiotic therapy for $P$. aeruginosa and MRSA is different from usual CAP therapy, it is important to recognize risk factors associated with these pathogens.

Risk factors for $P$. aeruginosa include structural lung diseases (bronchiectasis and COPD), nursing home residence, C-reactive protein less than $12.35 \mathrm{mg} / \mathrm{dl}$, prior use of oral steroids, antibiotic therapy within the last 90 days and malnutrition; chronic $P$. aeruginosa colonization in patients with bronchiectasis and COPD can be an important preliminary step to pneumonia too.

Recently, the study by Cillóniz et al. [48] in a cohort of 2023 patients with CAP reported that $4 \%$ of cases were caused by $P$. aeruginosa. Risk factors for this pathogen were male sex, chronic respiratory diseases, C-reactive protein less than $12.35 \mathrm{mg} / \mathrm{dl}$ and PSI risk class IV $-\mathrm{V}$. The only risk factor for MDR P. aeruginosa CAP was prior antibiotic treatment.

A Spanish study [49] analyzing 1519 patients with CAP found that PES pathogens (P. aeruginosa, Enterobacteriaceae extended spectrum b-lactamase-positive and MRSA) caused CAP in 6\% of patients and were associated with an increased risk of 30-day mortality. The authors proposed a score to assess the risk of pneumonia because of PES pathogens (Fig. 2).

The risk for MRSA is higher in patients with previous MRSA colonization, end-stage renal disease, contact sport participants, injection drug users, those living in crowded conditions, men who have sex with men and prisoners. Other risk factors include underlying lung diseases, recent influenza infection, previous antibiotic use, hospitalization in the previous 90 days and need for ICU admission $[50,51]$.

\section{WHAT IS THE RECOMMENDED EMPIRIC ANTIBIOTIC THERAPY IN SEVERE COMIMUNITY-ACQUIRED PNEUMONIA?}

Current international guidelines recommend the use of a macrolide or a respiratory fluoroquinolone in combination with a $\beta$-lactam for severe CAP $[1,52]$. Coverage for $P$. aeruginosa and MRSA should be administered depending on risk factors. However, there are not conclusive data regarding the superiority of $\beta$-lactam and macrolide (BL-M) compared to $\beta$-lactam and fluoroquinolones (BL-F) in the treatment of severe CAP.

A recent systematic review and meta-analysis [53"'] was performed on patients with severe CAP. Compared to patients treated by BL-F, patients receiving BL-M were discharged from hospital approximately 3 days earlier. However, ICU length of stay did not differ between the two groups. The overall mortality significantly differs between the two groups, with rates of 19 and $27 \%$ for the BL-M and the BL-F groups, respectively.

We recommend to follow the current international guidelines for the empiric therapy of severe CAP [1]. 
Emergencies in critical care

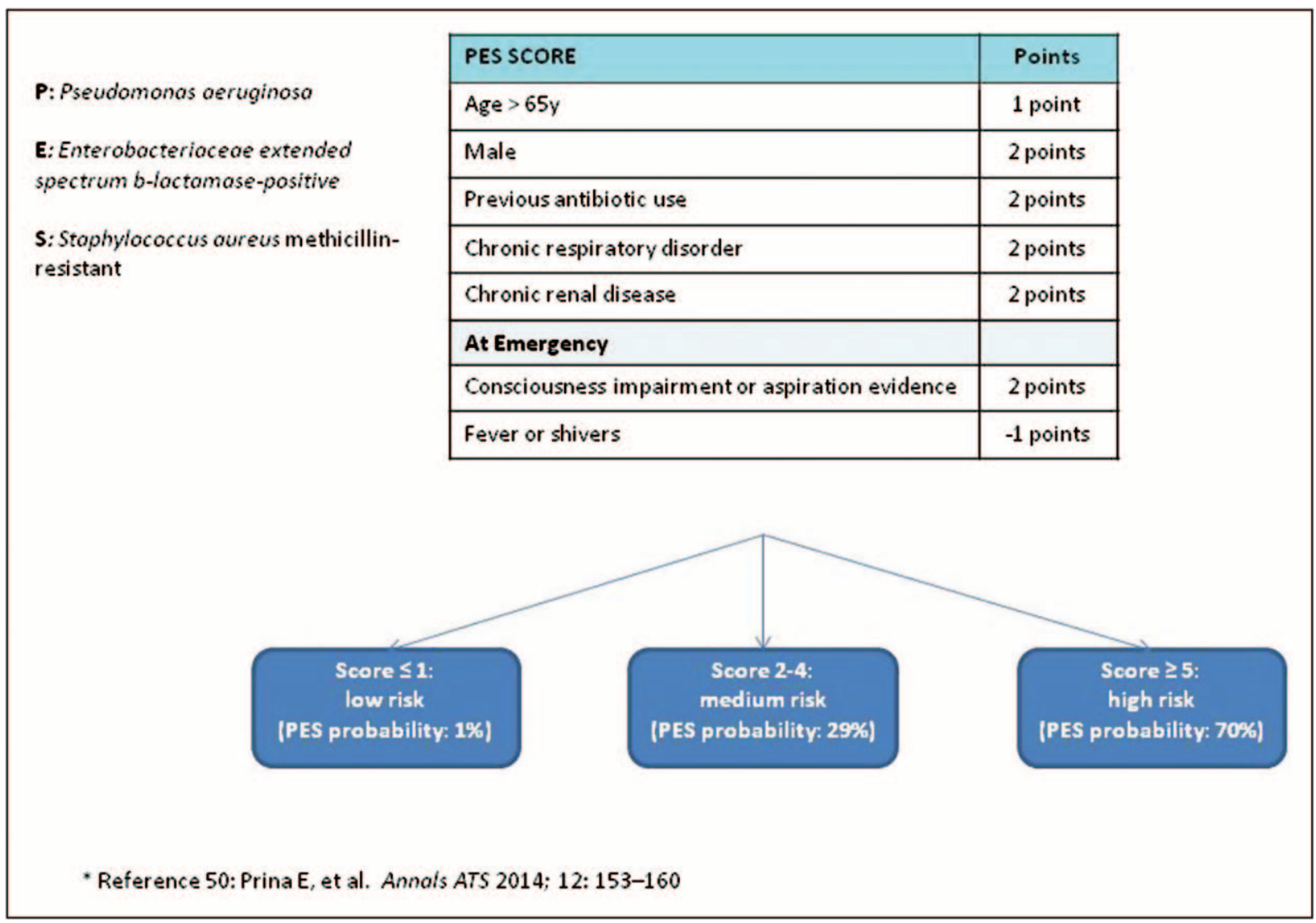

FIGURE 2. PES score.

\section{ADJUNCTIVE THERAPIES IN COMIMUNITY- ACQUIRED PNEUMONIA}

During the last decade, there has been an increasing interest in the protective role of adjunctive antiinflammatory therapies (mainly corticosteroids) in CAP, especially in severe cases.

\section{CORTICOSTEROIDS IN SEVERE COMMUNITY-ACQUIRED PNEUMONIA}

In 2015, an interesting randomized study found that in patients with severe CAP (according to ATS/IDSA criteria) and a high inflammatory response (initial levels of CRP $>15 \mathrm{mg} / \mathrm{dl}$ ), the use of low doses of corticosteroids compared with placebo decreased treatment failure with no difference in mortality [54] (Fig. 3).

A 2017 update [55] of a previously published review assessed the efficacy and safety of systemic corticosteroids, given as adjunct to antibiotic therapy, compared to placebo or no corticosteroids in the treatment of pneumonia. Corticosteroids reduced mortality and morbidity in adults with severe CAP; the number needed to treat for an additional beneficial outcome was 18 patients (95\% CI 12-49) to prevent one death. Corticosteroids, however, reduced morbidity but not mortality in adults and children with nonsevere CAP. Corticosteroid therapy was associated with more adverse events, especially hyperglycemia, but the harms did not seem to outweigh the benefits.

More recently, a systematic review analyzed all data from RCTs assessing the role of adjunctive corticosteroids in CAP [56]. The time to clinical stability and the hospital length of stay were reduced by approximately 1 day with corticosteroids. No significant effect on overall mortality was described. However, the authors found an increased risk for CAP-related rehospitalization and hyperglycemia with steroid treatment.

In conclusion, we recommend to use corticosteroids in the treatment of severe CAP cases with high inflammatory response (serum levels of C-reactive protein $\geq 15 \mathrm{mg} / \mathrm{dl}$ ) (Fig. 3).

\section{CORTICOSTEROIDS IN INFLUENZA PNEUMONIA}

A secondary analysis of a prospective cohort study investigated the clinical predictors associated with corticosteroids administration in critically ill patients with confirmed influenza pneumonia admitted to 148 ICUs in Spain [57']. The administration of corticosteroids in patients with severe 


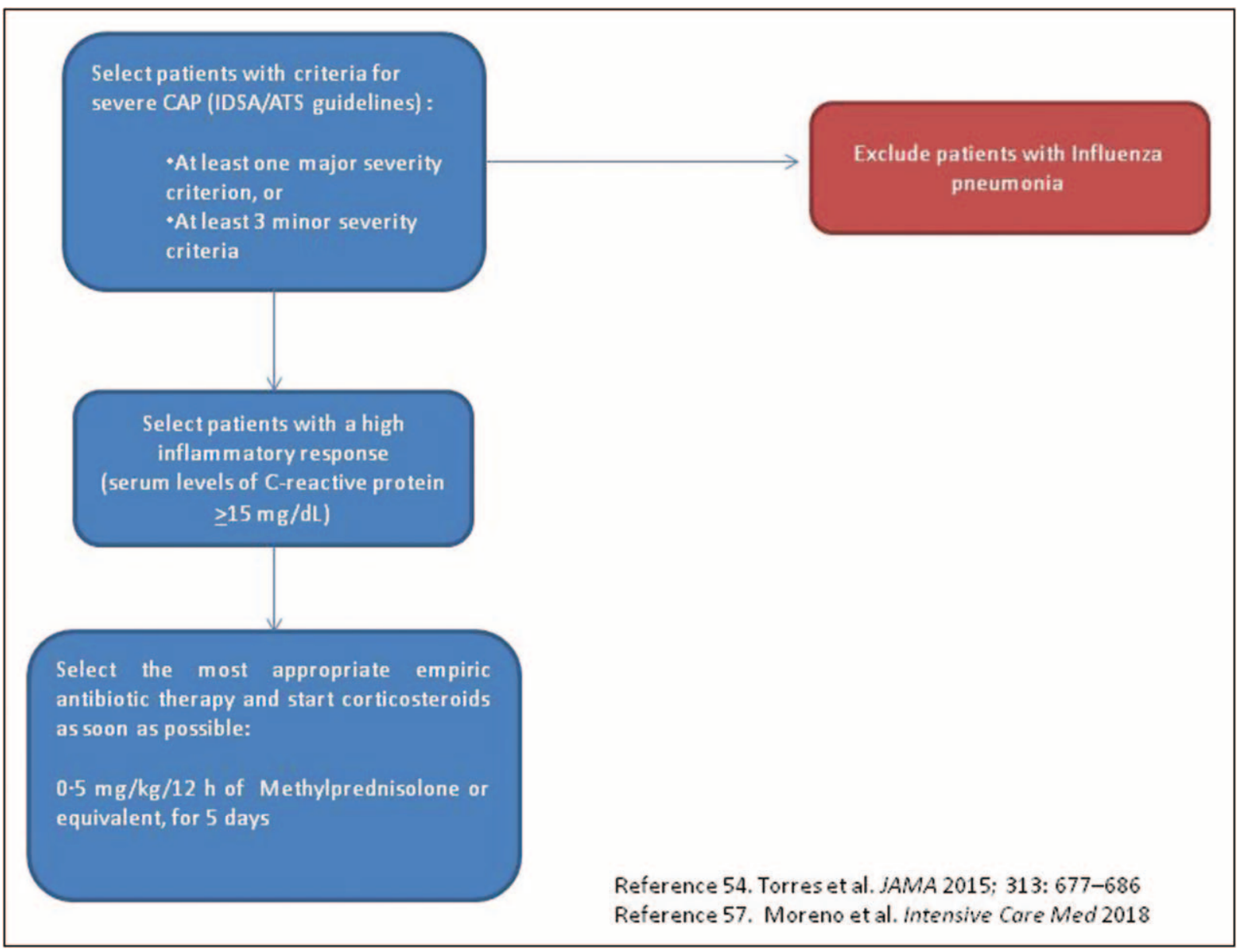

FIGURE 3. Algorithm of corticosteroids administration in severe CAP. CAP, community-acquired pneumonia.

influenza pneumonia was associated with increased ICU mortality. Consequently, the authors suggested not using corticosteroids as a coadjuvant therapy in patients with influenza pneumonia.

\section{CORTICOSTEROIDS IN SEPTIC SHOCK}

Two recent RCTs [10,11] evaluated the role of hydrocortisone in patients with septic shock, reporting conflicting results. In the ADRENAL trial [10], patients with septic shock undergoing mechanical ventilation were randomized to receive hydrocortisone (at a dose of $200 \mathrm{mg}$ per day) or placebo for 7 days or until death or discharge from the ICU. The continuous infusion of hydrocortisone in this population did not result in a lower 90-day mortality compared to placebo. The APROCCHSS trial [11] evaluated the effect of hydrocortisone-and-fludrocortisone compared to placebo in adult patients with septic shock. The authors reported that 90day all-cause mortality was lower among patients receiving hydrocortisone and fludrocortisone than among those receiving placebo.

\section{CONCLUSION}

CAP remains a significant cause of morbidity, mortality and health cost globally, despite the implementation of important measures that improve its management. The inclusion of biomarkers (independently or in combination) with severity scores will help to improve the early diagnosis of CAP, the prediction of its clinical severity and the early recognition of patients requiring ICU admission. Early appropriate empiric antibiotic therapy can increase survival and improve clinical outcomes, especially in severe CAP.

\section{Acknowledgements}

None.

\section{Financial support and sponsorship}

C.C. is the recipient of a Postdoctoral Grant (Strategic plan for research and innovation in health-PERIS 20162020). The funding source had no role in the article.

\section{Conflicts of interest}

All authors declare that they have no conflicts of interest. 


\section{REFERENCES AND RECOMMENDED READING}

Papers of particular interest, published within the annual period of review, have been highlighted as:

- of special interest

I. of outstanding interest

1. Mandell LA, Wunderink RG, Anzueto A, et al. Infectious Diseases Society of America, American Thoracic Society. Infectious Diseases Society of America/ American Thoracic Society consensus guidelines on the management of community-acquired pneumonia in adults. Clin Infect Dis 2007; 44(Suppl 2):S27-S72.

2. Eurich DT, Marrie TJ, Minhas-Sandhu JK, Majumdar SR. Ten-year mortality after community-acquired pneumonia. A prospective cohort. Am J Respir Crit Care Med 2015; 192:597-604.

3. Laporte L, Hermetet $\mathrm{C}$, Jouan $\mathrm{Y}$, et al. Ten-year trends in intensive care admissions for respiratory infections in the elderly. Ann Intensive Care 2018; 8:84.

4. Brown JD, Harnett J, Chambers R, Sato R. The relative burden of communityacquired pneumonia hospitalizations in older adults: a retrospective observational study in the United States. BMC Geriatr 2018; 18:92.

5. Montull B, Menéndez R, Torres A, et al. NAC Calidad Group. Predictors of severe sepsis among patients hospitalized for community-acquired pneumonia. PLoS One 2016; 11:e0145929.

6. Feldman C, Anderson R. Pneumonia as a systemic illness. Curr Opin Pulm Med 2018; 24:237-243.

7. Sinapidis D, Kosmas V, Vittoros V, et al. Progression into sepsis: an individualized process varying by the interaction of comorbidities with the underlying infection. BMC Infect Dis 2018; 18:242.

8. Cilloniz $C$ Ceccato $A$, San Jose $A$, Torres A. Clinical management of community acquired pneumonia in the elderly patient. Expert Rev Respir Med 2016

9. Ramirez JA, Wiemken TL, Peyrani $P$, et al. University of Louisville Pneumonia Study Group. Adults hospitalized with pneumonia in the United States: incidence, epidemiology \& mortality. Clin Infect Dis 2017; 65:1806-1812.

10. Venkatesh $B$, Finfer $S$, Cohen J, et al. ADRENAL Trial Investigators and the Australian-New Zealand Intensive Care Society Clinical Trials Group. Adjunctive glucocorticoid therapy in patients with septic shock. N Engl J Med 2018; 378:797-808.

11. Annane $D$, Renault $A$, Brun-Buisson $C$, et al. Hydrocortisone plus fludrocortisone for adults with septic shock. N Engl J Med 2018; 378:809-818.

12. Storms $A D$, Chen J, Jackson $L A$, et al. Rates and risk factors associated with hospitalization for pneumonia with ICU admission among adults. BMC Pulm Med 2017; 17:208.

13. Cilloniz C, Ferrer M, Liapikou A, et al. Acute respiratory distress syndrome in mechanically ventilated patients with community-acquired pneumonia. Eur Respir J 2018; 51:; pii: 1702215

14. Lim WS, van der Eerden MM, Laing $R$, et al. Defining community acquired pneumonia severity on presentation to hospital: an international derivation and validation study. Thorax 2003; 58:377-382.

15. Torres A, Garcia-Vidal C. Mortality of CAP reduced in the UK: is this enough? Thorax 2016; 71:979-980.

16. Ferrer $M$, Travierso $C$, Cilloniz $C$, et al. Severe community-acquired pneumonia: characteristics and prognostic factors in ventilated and nonventilated patients. PLoS One 2018; 13:e0191721.

17. Williams $\mathrm{JM}$, Greenslade $\mathrm{JH}, \mathrm{Chu} \mathrm{KH}$, et al. Utility of community-acquired pneumonia severity scores in guiding disposition from the emergency department: intensive care or short-stay unit? Emerg Med Australas 2018; 30:538-546.

18. Cilli $A$, Cakin $O$, Aksoy $E$, et al. Acute cardiac events in severe communityacquired pneumonia: a multicenter study. Clin Respir J 2018; 12:2212-2219.

19. Fine MJ, Auble TE, Yealy DM, et al. A prediction rule to identify low-risk patients with community-acquired pneumonia. N Engl J Med 1997; 336:243-250.

20. Ranzani OT, Prina E, Menéndez R, et al. New sepsis definition (sepsis-3) and

- community-acquired pneumonia mortality. A validation and clinical decisionmaking study. Am J Respir Crit Care Med 2017; 196:1287-1297.

Comparison between different clinical scores. PSI to predict mortality.

21. Charles PGP, Wolfe $R$, Whitby $M$, et al. Australian Community-Acquired Pneumonia Study Collaboration, Grayson M.L., SMART-COP: a tool for predicting the need for intensive respiratory or vasopressor support in community-acquired pneumonia. Clin Infect Dis 2008; 47:375-384.

22. Lim HF, Phua J, Mukhopadhyay A, et al. IDSA/ATS minor criteria aid preintensive care unit resuscitation in severe community-acquired pneumonia. Eur Respir J 2014; 43:852-862.

23. Simplification of the IDSA/ATS criteria for severe CAP using meta-analysis and observational data. - PubMed - NCBI [Internet]. [Accessed 20 August 2018]. Available from: https://www.ncbi.nlm.nih.gov/pubmed/ ?term=Simplification+of+the+IDSA\%2FATS+criteria+for+severe+CAP+using+meta-analysis+and+observational+data.
24. Chalmers JD, Singanayagam A, Hill AT. Predicting the need for mechanical ventilation and/or inotropic support for young adults admitted to the hospital with community-acquired pneumonia. Clin Infect Dis 2008; 47 : $1571-1574$

25. Marti C, Garin N, Grosgurin O, et al. Prediction of severe community-acquired pneumonia: a systematic review and meta-analysis. Crit Care 2012; 16:R141.

26. Bermejo-Martin JF, Cilloniz $C$, Mendez $R$, et al. NEUMONAC group. Lym-

- phopenic community acquired pneumonia (L-CAP), an immunological phenotype associated with higher risk of mortality. EBioMedicine 2017 $24: 231-236$.

Interesting study about the 'lymphopenic CAP profile'.

27. Bermejo-Martin JF, Almansa R, Martin-Fernandez M, et al. Immunologica profiling to assess disease severity and prognosis in community-acquired pneumonia. Lancet Respir Med 2017; 5:e35-e36.

28. Luo $Q$, Ning $P$, Zheng $Y$, et al. Serum suPAR and syndecan- 4 levels predict severity of community-acquired pneumonia: a prospective, multicentre study. Crit Care 2018; 22:15

29. Zhou $\mathrm{H}$, Guo $\mathrm{S}$, Lan $\mathrm{T}$, et al. The risk stratification and prognostic prediction value of procalcitonin and clinical severity scores on patients with communityacquired pneumonia in emergency department: Prediction value of procalcitonin and severity scores for CAP. Am J Emerg Med 2018. Mar 21. pii: S0735-6757(18)30242-0.

30. Legramante JM, Mastropasqua $M$, Susi $B$, et al. Prognostic performance of MR-pro-adrenomedullin in patients with community acquired pneumonia in the emergency department compared to clinical severity scores PSI and CURB. PLoS One 2017; $12:$ e0187702.

31. Gilbert DN. Role of procalcitonin in the management of infected patients in the intensive care unit. Infect Dis Clin North Am 2017; 31:435-453.

32. Julián-Jiménez A, González Del Castillo J, Candel FJ. Usefulness and prog nostic value of biomarkers in patients with community-acquired pneumonia in the emergency department. Med Clin (Barc) 2017; 148:501-510.

33. Keramat $F$, Ghasemi Basir HR, Abdoli $E$, et al. Association of serum procalcitonin and C-reactive protein levels with CURB-65 criteria among patients with community-acquired pneumonia. Int J Gen Med 2018; 11:217-223.

34. Spoorenberg SMC, Vestjens SMT, Voorn GP, et al. Ovidius study group. Course of SP-D, YKL-40, CCL18 and CA 15-3 in adult patients hospitalised with community-acquired pneumonia and their association with disease severity and aetiology: a posthoc analysis. PLoS One 2018; 13:e0190575.

35. Khan $F$, Owens MB, Restrepo $M$, et al. Tools for outcome prediction in patients with community acquired pneumonia. Expert Rev Clin Pharmaco 2017; 10:201-211.

36. Self WH, Wunderink RG, Jain $S$, et al. Etiology of Pneumonia in the Community (EPIC) Study Investigators. Procalcitonin as a marker of etiology in adults hospitalized with community-acquired pneumonia. Clin Infect Dis 2018; 66:1640-1641.

PCT to guide antibiotic therapy: no predictive.

37. Huang DT, Yealy DM, Filbin MR, et al. ProACT Investigators. Procalcitoninguided use of antibiotics for lower respiratory tract infection. N Engl J Med 2018; 379:236-249.

38. Chu DC, Mehta AB, Walkey AJ. Practice patterns and outcomes associated with procalcitonin use in critically ill patients with sepsis. Clin Infect Dis 2017 64:1509-1515.

39. Schuetz $P$, Wirz $Y$, Sager $R$, et al. Procalcitonin to initiate or discontinue

antibiotics in acute respiratory tract infections. Cochrane Database Syst Rev 2017; 10:CD007498

PCT to guide antibiotic therapy: predictive.

40. McCluskey SM, Schuetz P, Abers MS, et al. Serial procalcitonin as a predicto

- of bacteremia and need for intensive care unit care in adults with pneumonia, including those with highest severity: a prospective cohort study. Open Forum Infect Dis 2017; 4:ofw238.

PCT as a predictive tool.

41. Kim MW, Lim JY, Oh SH. Mortality prediction using serum biomarkers and various clinical risk scales in community-acquired pneumonia. Scand J Clin Lab Invest 2017; 77:486-492.

42. Shi SJ, Li H, Liu M, et al. Mortality prediction to hospitalized patients with influenza pneumonia: PO2 /FiO2 combined lymphocyte count is the answer. Clin Respir J 2017; 11:352-360.

43. Cataudella E, Giraffa CM, Di Marca S, et al. Neutrophil-to-lymphocyte ratio: an emerging marker predicting prognosis in elderly adults with communityacquired pneumonia. J Am Geriatr Soc 2017; 65:1796-1801.

44. Camon $S$, Quiros $C$, Saubi N, et al. Full blood count values as a predictor of poor outcome of pneumonia among HIV-infected patients. BMC Infect Dis 2018; 18:189.

45. Brendish NJ, Malachira AK, Armstrong L, et al. Routine molecular point-of-care

- testing for respiratory viruses in adults presenting to hospital with acute respiratory illness (ResPOC): a pragmatic, open-label, randomised controlled trial. Lancet Respir Med 2017; 5:401-411.

Role of POCT in pneumonia.

46. Restrepo Ml, Babu BL, Reyes LF, et al. Burden and risk factors for Pseudomonas aeruginosa community-acquired pneumonia: a multinational point prevalence study of hospitalised patients. Eur Respir J 2018; 52:; pii: 1701190 
47. Cillóniz $C$, Rodríguez-Hurtado D, Nicolini A, Torres A. Clinical approach to community-acquired pneumonia. J Thorac Imaging 2018; 33:273-281.

48. Cillóniz $\mathrm{C}$, Gabarrús $\mathrm{A}$, Ferrer $\mathrm{M}$, et al. Community-acquired pneumonia due to multidrug and non-multidrug resistant Pseudomonas aeruginosa. Chest $2016 ; 150: 415-425$

49. Prina E, Ranzani OT, Polverino E, et al. Risk factors associated with potentially antibiotic-resistant pathogens in community-acquired pneumonia. Ann Am Thorac Soc 2014; $12: 153-160$.

50. Aliberti S, Reyes LF, Faverio P, et al. GLIMP investigators. Global initiative for meticillin-resistant Staphylococcus aureus pneumonia (GLIMP): an international, observational cohort study. Lancet Infect Dis 2016; 16:1364-1376.

51. Torres Bonafonte $\mathrm{OH}$, Gil Olivas $\mathrm{E}$, Pérez Macho E, et al. [Predictors of drugresistant pathogens in community-onset pneumonia: Are factors considered in health-care-associated pneumonia useful in the emergency department?]. Emergencias 2017; 29:306-312.

52. Lim WS, Baudouin SV, George RC, et al. Pneumonia Guidelines Committee of the BTS Standards of Care Committee. BTS guidelines for the management of community acquired pneumonia in adults: update. Thorax 2009; 64(Suppl 3):iii1-iii55.
53. Lee JH, Kim HJ, Kim YH. Is $\beta$-lactam plus macrolide more effective than $\beta$ -

-1 lactam plus fluoroquinolone among patients with severe community-acquired pneumonia?: a systemic review and meta-analysis. J Korean Med Sci 2017; $32: 77-84$

Treatment of severe CAP: $\beta$-lactam and macrolide versus $\beta$-lactam and respiratory fluoroquinolone.

54. Torres $A$, Sibila $O$, Ferrer $M$, et al. Effect of corticosteroids on treatment failure among hospitalized patients with severe community-acquired pneumonia and high inflammatory response: a randomized clinical trial. JAMA 2015; 313:677-686.

55. Stern A, Skalsky K, Avni T, et al. Corticosteroids for pneumonia. Cochrane Database Syst Rev 2017; 12:CD007720.

56. Briel M, Spoorenberg SMC, Snijders D, et al. Ovidius Study Group, Capisce Study Group, STEP Study Group. Corticosteroids in patients hospitalized with community-acquired pneumonia: systematic review and individual patien data metaanalysis. Clin Infect Dis 2018; 66:346-354.

57. Moreno G, Rodriguez A, Reyes LF, et al. on Behalf GETGAG Study Group.

- Corticosteroid treatment in critically ill patients with severe influenza pneumonia: a propensity score matching study. Intensive Care Med 2018; 44:1470-1482.

Corticosteroids in influenza pneumonia. 


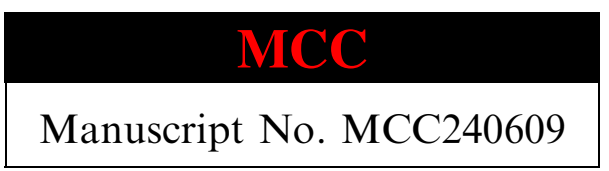

\section{Current Opinion in Critical Care \\ Typeset by Thomson Digital \\ for Wolters Kluwer}

Dear Author,

During the preparation of your manuscript for typesetting, some queries have arisen. These are listed below. Please check your typeset proof carefully and mark any corrections in the margin as neatly as possible or compile them as a separate list. This form should then be returned with your marked proof/list of corrections to the Production Editor.

\section{QUERIES: to be answered by AUTHOR/EDITOR?}

\begin{tabular}{|c|c|c|}
\hline QUERY NO. & QUERY DETAILS & RESPONSE \\
\hline$<\mathrm{AQ} 1>$ & $\begin{array}{l}\text { As per style, the short title/running head } \\
\text { can have a maximum of } 65 \text { characters } \\
\text { including spaces and author names, and } \\
\text { abbreviations/acronyms only as } \\
\text { exceptions. Please check the suggested } \\
\text { short title, "Community-acquired } \\
\text { pneumonia Cillóniz et al." for } \\
\text { appropriateness. }\end{array}$ & \\
\hline$<\mathrm{AQ} 2>$ & $\begin{array}{l}\text { Please confirm whether surnames/family } \\
\text { names (red) have been identified } \\
\text { correctly in the author byline. }\end{array}$ & \\
\hline$<\mathrm{AQ} 3>$ & $\begin{array}{l}\text { Please check affiliation and } \\
\text { correspondence for correctness. }\end{array}$ & \\
\hline$<\mathrm{AQ} 4>$ & $\begin{array}{l}\text { Please check all section headings for } \\
\text { correctness. }\end{array}$ & \\
\hline$<\mathrm{AQ} 5>$ & $\begin{array}{l}\text { Please provide the full form of COPD, } \\
\text { HCAP, RCT. }\end{array}$ & \\
\hline$<\mathrm{AQ} 6>$ & $\begin{array}{l}\text { As the following references are outside } \\
\text { the review period, bullets and } \\
\text { annotations have been deleted as per } \\
\text { style. Refs. }[1,48,54] \text {. }\end{array}$ & \\
\hline$<\mathrm{AQ} 7>$ & $\begin{array}{l}\text { Please provide the volume number and } \\
\text { page range in } 8,29 \text {. }\end{array}$ & \\
\hline$<\mathrm{AQ} 8>$ & Please provide the page range in 13,46 . & \\
\hline
\end{tabular}

